\title{
Towards a Passive Adaptive Planar Foot with Ground Orientation and Contact Force Sensing for Legged Robots
}

\section{Conference Paper}

Author(s):

Käslin, Roman; Kolvenbach, Hendrik; Paez, Laura; Lika, Klajd; Hutter, Marco (D)

Publication date:

2018-10

Permanent link:

https://doi.org/10.3929/ethz-b-000276595

Rights / license:

$\underline{\text { In Copyright - Non-Commercial Use Permitted }}$

Originally published in:

https://doi.org/10.1109/IROS.2018.8593875 


\title{
Towards a Passive Adaptive Planar Foot with Ground Orientation and Contact Force Sensing for Legged Robots
}

\author{
Roman Käslin, Hendrik Kolvenbach, Laura Paez, Klajd Lika and Marco Hutter
}

\begin{abstract}
Adapting to the ground enables stable footholds in legged locomotion by exploiting the structure of the terrain. On that account, we present a passive adaptive planar foot with three rotational degrees of freedom that is lightweight and thus suited for highly dynamic legged robots. Its low laying pivot joint provides high stability towards kinking. Information about the relative foot sole pose, and accordingly, the ground orientation is gathered by inertial measurement units (IMUs) placed on the foot sole and the shank. A complementary filter is presented that fuses these orientation estimates with an angular encoder to obtain a drift-free relative foot sole pose. The passive adaptive planar foot has been tested and compared to the classical point foot design on a variety of terrains and shows superior traction performance, especially on compressible soils. Being mounted on the quadrupedal robot ANYmal, the foot provides a reliable contact detection based on the fusion of the built-in 6-axis force/torque transducer and the IMUs. This allows to walk and trot on uneven terrain, loose soils, as well as climbing up a ramp and stairs while keeping the entire foot sole in ground contact all the time.
\end{abstract}

\section{INTRODUCTION}

Legged robots have superior abilities to overcome rough terrain compared to wheeled systems; they can interact with the environment at dedicated contact points and are nevertheless capable of carrying a substantial payload. One example of such a robot is the quadruped ANYmal [1]. It is designed to operate autonomously in challenging environments, e.g. in search and rescue scenarios or remote industrial inspection tasks [2]. Consequently, the terrain on which the robot operates varies broadly depending on the application.

Most quadruped robots have ball-shaped feet like BigDog [3], LittleDog [4], MIT Cheetah [5] or ANYmal [1]. Ballshaped feet designs are used on quadrupedal robots because they provide sufficient points of contact and thus do not need to exert torques on the ground. The ball-shaped design allows using the feet in nearly every orientation with respect to the ground. However, the only ground adaption is provided by a deformation of the hemisphere and is thus limited to a small area around the point of contact. On ANYmal and in case of hard and flat surfaces, the contact area is typically $8 \mathrm{~cm}^{2}$. This limits the operating capabilities, especially on compressible ground [6]. Additionally, the current design of the point foot does not give any information on a potential inclination of the terrain at the foothold [7] or the contact point itself. Another disadvantage of the point foot design is

This work was supported by the Swiss National Science Foundation through the National Center of Competence in Research (NCCR) Robotics, the European Space Agency (ESA) and Airbus DS in the framework of the Network Partnering Initiative 481-2016 and the EU project THING.

All the authors are with the Robotic Systems Lab (RSL) at ETH Zurich, Switzerland hendrik. kolvenbach@mavt.ethz.ch

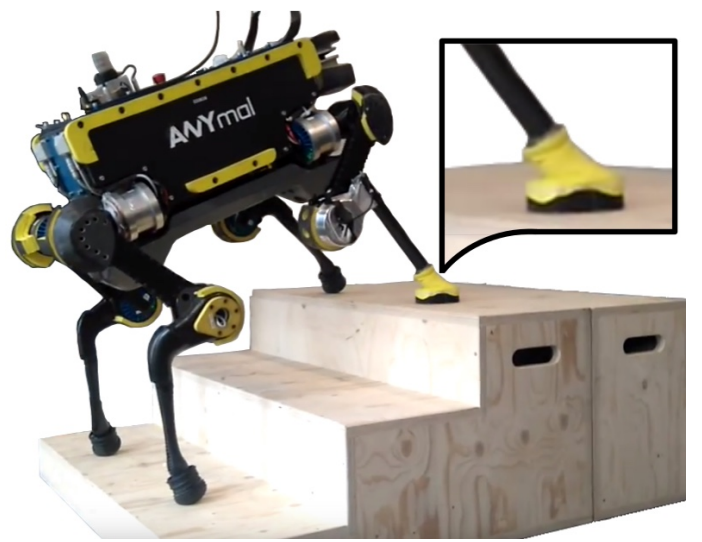

Fig. 1: The adaptive foot allows the quadrupedal robot ANYmal to adapt to the geometry of the ground and gather information about the ground inclination. It features a larger contact area than comparable ball-shaped feet providing better impact absorption and force distribution. The measured contact forces allow for reliable contact detection and permit to optimize the force distribution among the feet.

the moving and thus non-constant point of contact while the robot is moving, which additionally makes contact force and inclination determination, especially on granular media, very difficult.

In this paper, a passive adaptive planar foot is introduced. The goal is to improve the robot's ability to cope with a broad range of terrains by passively adapting with three rotational degrees of freedom to the ground. The adaptability allows for a planar contact and thus increased contact surface that distributes the forces on a larger area. This extends the operating capabilities of the robot on inclined and compressible terrains. Additionally, the sensors allow for direct contact force and ground orientation measure which increases the perceptional capabilities.

\section{A. Related Work}

Adaptive feet are used by legged animals to perceive the world and adapt to the ground [8], [9]. An impressive example is the climbing capabilities of mountain goats whose hoofs consist of an outer hard rim and an inner soft pad that is analyzed in the work of Abad et al. [8]. The authors also show, that slipping a bio-inspired adaptive hoof requires about three times more work than slipping a comparable ball shaped foot. In rehabilitation engineering, the design of feet prostheses is a large research area and various prosthetic device featuring passive [10] and active compliance [11], [12], [13] are developed. All the active prostheses feature series and/or parallel elastics besides the powered ankle motion to 
allow for impact absorption and to enable a natural walking gait.

Passively adapting feet can be used for the design of bipedal robots when only dynamic stability is required, like for example the ATRIAS robot [14]. On the other hand, most biped robots use feet with actuated dorsiflexion/plantarflexion and inversion/eversion as the Honda ASIMO robot [15], HRP3 [16], Lola [17], KHR-3 [18] and WALK-MAN [19]. Mainly 6-axes force/torque transducers are used to measure the forces and torques acting on the feet of biped robots.

Some feet imitate the damping and the windlass mechanism of human feet, such as the design of the compliant SoftFeet [20] or the design by Narioka et al. [21]. In the design of Fondahl et al. [22], the rear feet of an robotic ape feature actuated dorsiflexion/plantarflexion and inversion/eversion with included force distribution measurements by an array of force sensitive resistors combined with a 6-axis force/torque transducer.

In order to measure the orientation of the ankle joint, IMUs can be used. A commonly used algorithm, which is broadly used on quadrocopters, is the gradient descent algorithm of Madgwick [23]. Additionally, Kalman filter implementations, as the ones proposed by [24], [25], and efficient complementary filters, as the one of Valenti et al. [26] exist.

\section{B. Paper Outline}

The mechanical design of the adaptive foot and the built in electronic components are described in Sec. II. Sec. III introduces the implementation of the sensor fusion and contact estimation algorithms. Subsequently, the test setup is presented in Sec. IV, and the results are discussed in Sec. V. Finally, the work is concluded in Sec. VI.

\section{DESIGN}

The adaptive foot is designed to ensure traction on hard surfaces as well as compressible soils. It also measures the ground orientation defined as the plane in contact with the ground (i.e. below the foot sole). To facilitate these characteristics, it has a planar foot sole that provides a large area during ground contact. The foot sole should be laying flat on the ground during the entire gait cycle of $40^{\circ}$ dorsiflexion and $10^{\circ}$ inversion/eversion. With an additional possible inclination of the terrain of $\pm 25^{\circ}$, the range of motion (ROM) for the ground compliance is set to $\pm 45^{\circ}$ around pitch and $\pm 30^{\circ}$ around roll.

Furthermore, since each of the four legs only allows for hip abduction/adduction, hip flexion/extension and knee flexion/extension, the foot also includes a ROM of $\pm 20^{\circ}$ around yaw to prevent the foot from slipping while turning. With a weight of $380 \mathrm{~g}$ the foot is slightly heavier than the previous point foot design, which is $325 \mathrm{~g}$, but designed lightweight to minimize inertia with regard to the hip and knee actuators.

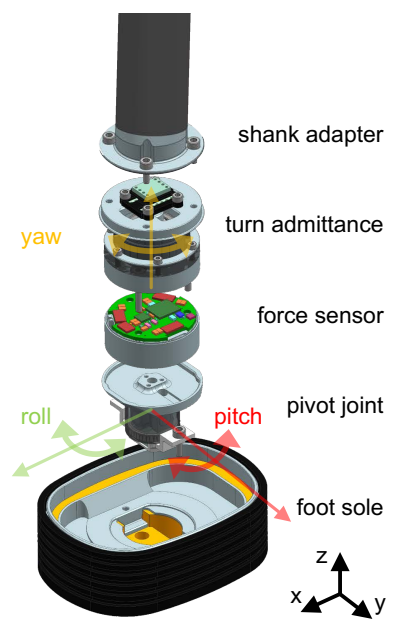

(a) sub-assemblies of the foot

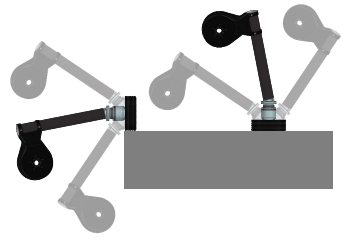

(b) foot sole usage

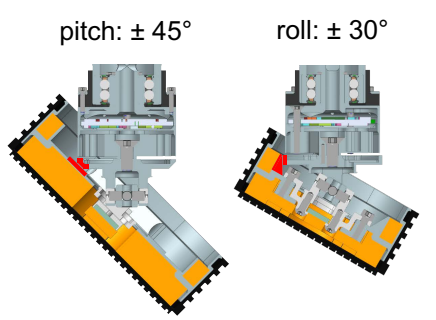

(c) end stop for roll \& pitch
Fig. 2: The different sub-assemblies of the foot and the degrees of freedom around roll, pitch and yaw are shown in (a). The foot can also be used on its side since the point of rotation lays within the foot sole rim (b). The range of motion is limited by a physical end stop highlighted in (c).

\section{A. Mechanical Structure}

The physical arrangement of components shown in Fig. $2 \mathrm{a}$ is mainly determined through the demand to minimize the swinging motion of the foot sole during the swing phase of the leg. Therefore, the pivot joint featuring pitch and roll flexibility is placed as close to the ground as possible. Having the point of rotation within the supporting polygon of the rim around the foot sole enables the use of the foot on its side for special maneuvers like standing up, as shown in Fig. 2 b. The force sensor is placed immediately above the pivot joint to minimize the lever arm, and hence the torque acting on the transducer in case the forces appear in $\mathrm{x} / \mathrm{y}$-direction. Going further up, the turn admittance around yaw and the shank adapter follow. The entire foot is sealed against water and dust by an outer shell in between the exchangeable foot sole and the shank adapter. The foot is dimensioned for peak loads up to $1000 N$ in z-direction and up to $500 N$ in $\mathrm{x} / \mathrm{y}$-direction.

1) Foot Sole: The foot sole made of a corrugated NR/SBR rubber pad of Shore A65 has an outer size of roughly $10 \mathrm{~cm}$ x $7 \mathrm{~cm}$ featuring $50 \mathrm{~cm}^{2}$ of the contact area, and is damped by $12 \mathrm{~mm}$ of memory foam (Poron XRD). The same foam is used on the previous point foot and the overall stiffness is inspired by the haptic feeling of a goat foot sole. The foot sole pattern consists of 150 small rubber studs with a height of $1.5 \mathrm{~mm}$ each. It is surrounded by a finned outer rubber rim of NR/SBR with Shore A65 that is supported by an aluminum rim serving as a limitation for the deformation of the foot sole. Additionally, it provides footholds on all sides perpendicular to the foot sole, as shown in Fig. 2b. The aluminum ring is restricted in $\mathrm{z}$-direction and damped in $\mathrm{x}$ and $y$-direction towards an inner basin that collects all the forces and provides the interface to the pivot joint.

2) Pivot Joint: The pivot joint features a universal joint whose components are made of steel and titanium to provide the necessary robustness while minimizing the size and weight. 
It is surrounded by an Ester Polyurethane rubber tube of Shore A50 that provides the compliance to reset the foot to its initial position after deflection.

The orientation of the foot sole is measured by an IMU (IMU1) placed below the universal joint within the bracket used for mounting the pivot joint to the foot sole. To minimize the motion that the IMU cables have to undergo, they are routed through the center of the joint. The circular plate of the upper fork of the pivot joint provides the end stop for the pivoting motion around pitch and roll highlighted in Fig. 2c. To reduce peak loads when reaching the end stop, the touching surfaces on the basin of the foot sole are damped.

3) Force Sensor: A custom, in-house developed 6-axis force/torque sensor is placed in between the pivot joint and the turn admittance to measure the forces acting on the foot. It consists of a force sensing element with strain gauges and a printed circuit board (PCB), specified in Sec. II-B. The custom sensor allows for an integrated and lightweight design.

4) Turn Admittance: Yaw motion is allowed by an angular ball bearing that is capable of handling the high axial forces of the vertical impacts. The bearing is mounted on a hollow axle that provides space for cable routing on the inside. The bearing housing is connected with a silicon rubber flange of Shore A50 to the lower plate of the axle on the outside. This rubber provides the compliance and limits the turning motion. On the top of the axle a diametric magnet is placed, which is read out by an angular encoder fixated to the bearing housing. Above the angular encoder a second IMU (IMU2) is mounted to measure the orientation of the shank.

5) Shank: A shank adapter is glued to the carbon tube connecting to the knee joint. The processing board that gathers all the sensor data is placed within the carbon tube.

6) Sealing: Between the outer rubber ring of the foot sole and the shank adapter, a commercial latex balloon seals the inner part of the foot towards the environment.

\section{B. Electrical Components}

The electronics of the foot consists of two IMUs, an angular encoder, a force sensor and a microcontroller board, as shown in Fig. 3. The IMUs and the angular encoder are connected to the microcontroller via serial peripheral interface bus (SPI), whereas the force sensor is connected via universal asynchronous receiver/transmitter (UART). The IMUs and the angular encoder are read out with $4 \mathrm{kHz}$ and force measurements are obtained with $400 \mathrm{~Hz}$. The microcontroller board is powered and connected to the robot via USB.

InvenSense ICM-20608-G IMUs are used due to their fast accelerometer speed $(4 \mathrm{kHz})$. As angular encoder, the AMS AS5048A with 14-bit full turn resolution and an accuracy of $0.06^{\circ}$ is installed. The custom 6-axes force/torque features a PCB with analog-to-digital converters (ADCs) and a microcontroller that processes the analog signals of the strain gages. As microcontroller development board a Teensy 3.6 with $180 \mathrm{M} \mathrm{Hz}$ clock speed is used on account of its small size and its high processing power.

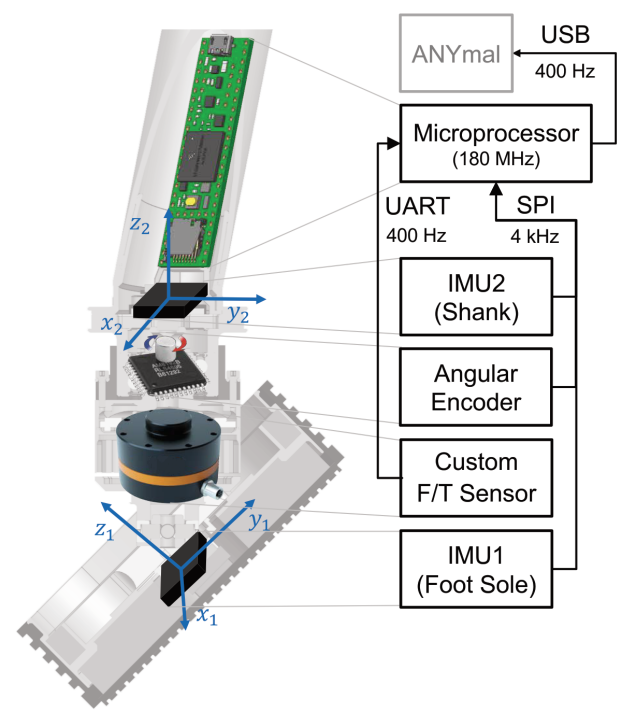

Fig. 3: The foot contains two inertial measurement units (IMUs), one in the foot sole and one in the shank to measure the corresponding orientations. It comprises a force/torque transducer to measure the loads acting on the foot and an angular encoder to couple both IMUs. All these sensors are connected to the microcontroller board housed within the shank.

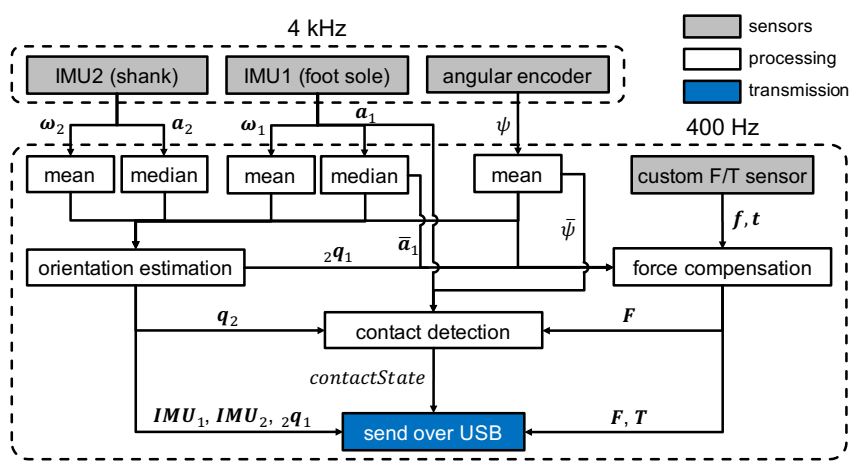

Fig. 4: The data processing on the microcontroller board operates at $400 \mathrm{~Hz}$. Therefore, the data obtained with $4 k H z$ is filtered before computing the orientation estimate. Subsequently, the measured forces are compensated for inertial forces and the contact state is estimated. Finally, all data is sent over USB to the robot.

\section{IMPLEMENTATION}

The gathered sensor data is fused on the microcontroller board inside the foot. Therefore, a $4 k H z$ interval timer on the Teensy board calls the read-out of the IMUs and the angular encoder every iteration and the force sensor every 10th iteration. Similarly, the processed data is sent via USB every 10 th iteration.

In parallel, the data processing shown in Fig. 4 operates on the Teensy board in a $400 \mathrm{~Hz}$ loop. A median filter is used for the more outlier-prone accelerometer data $a_{i}$ and a mean filter for gyroscope values $\omega_{i}$ and encoder data $\psi$ of the last ten measurements. The orientation of the foot sole, the shank and the relative orientation in between are estimated as described in subsection III-A. The force measurements are compensated for inertial forces (subsection III-B) and it is estimated whether the foot is in ground contact (subsection III-C). 


\section{A. Foot Sole Pose Estimation}

A sensor fusion algorithm is implemented to estimate the relative foot sole pose with regard to the shank. Since the joint axis of the universal joint featured in our design is not fixed, the proposed method of Seel et al. [27] cannot be applied. Instead, a new algorithm that fuses the accelerometer and gyroscope data of both IMUs together with the encoder measurement to obtain a stable and drift free estimate is introduced. To do so, the complementary filter of Valenti et al. [26], is expanded to include two IMUs which are coupled with the encoder measurement, as shown in Fig. 5.

The state of the filter at time $k$ is defined as the combination of the orientation estimates of both IMUs in the form of quaternions.

$$
\boldsymbol{x}_{k}=\left[\boldsymbol{q}_{1, k}, \boldsymbol{q}_{2, k}\right]^{T} \text { with } \boldsymbol{q}_{i, k}=\left[q_{i 0, k}, q_{i 1, k}, q_{i 2, k}, q_{i 3, k}\right]^{T}
$$

The prediction and accelerometer correction of the orientation estimate for both IMUs are identical with the implementation of Valenti et al. [26]. The bias $\boldsymbol{b}_{i}$ is predefined at startup and gets subtracted from the measured gyroscope data before computing the quaternion derivative $\dot{\boldsymbol{q}}_{i, k}^{(p)}$. The prediction step is completed by integrating over the time passed since the last update, addition to the previous estimate $\boldsymbol{q}_{i, k-1}$ and renormalization to unit length.

For the accelerometer correction and the subsequent coupling of both IMUs for every time step $k$ the subscript $k$ is omitted for better readability. The predicted orientation $\boldsymbol{q}_{i}^{(p)}$ is used to rotate the normalized acceleration measurements that results in the predicted gravity vector $\boldsymbol{g}_{i}^{(p)}=\left[g_{i x}, g_{i y}, g_{i z}\right]^{T}$. The quaternion $\Delta \boldsymbol{q}_{i}$ by which $\boldsymbol{g}_{i}^{(p)}$ has to be rotated to be aligned with the actual gravity vector $\boldsymbol{g}=[0,0,1]^{T}$ is then computed as

$$
\Delta \boldsymbol{q}_{i}=\left[\sqrt{\frac{g_{i z}+1}{2}}, \frac{g_{i y}}{\sqrt{2\left(g_{i z}+1\right)}}, \frac{-g_{i x}}{\sqrt{2\left(g_{i z}+1\right)}}, 0\right]^{T} .
$$

Subsequently, the correction quaternion is computed as a spherical linear interpolation (SLERP) [28] of $\boldsymbol{q}_{I}=$ $[1,0,0,0]^{T}$ and $\Delta \boldsymbol{q}_{i}$ with gain $\alpha_{g}$

$$
\boldsymbol{q}_{i}^{\text {corr }}=\frac{\sin \left(\left(1-\alpha_{g}\right) \Delta q_{i 0}\right)}{\sin \left(\Delta q_{i 0}\right)} \boldsymbol{q}_{I}+\frac{\sin \left(\alpha_{g} \cdot \Delta q_{i 0}\right)}{\sin \left(\Delta q_{i 0}\right)} \Delta \boldsymbol{q}_{i}
$$

and applied on the predicted quaternions with subsequent renormalization to unit length. Instead of implementing an adaptive gain as Valenti et al. [26] the accelerometer update uses an experimentally determined fixed gain of $\alpha_{g}=0.1$ and is only executed if the norm of the measured acceleration of both IMUs does not differ from gravity $G=9.81 \mathrm{~m} / \mathrm{s}^{2}$ by more than ten percent, given by

$$
|| \overline{\boldsymbol{a}}_{i}|-G|<0.1 \cdot G \quad \forall i
$$

The coupling of both IMUs with the encoder measurements is solved analogously to the acceleration update. First, the Euler angle around yaw of the relative foot sole pose is

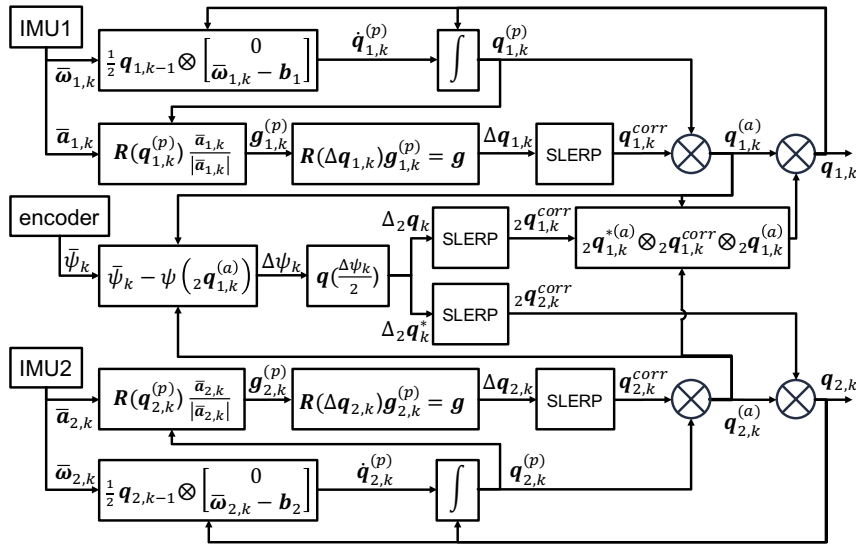

Fig. 5: For both IMUs a standard complementary filter integrates the gyroscope data as prediction and corrects it by aligning accelerometer data to the gravity vector. To couple both orientation estimates the interjacent yaw angle is compared to the measurement of the angular encoder and a correction is applied on both estimates.

determined. The foot sole pose (frame 1) relative to the shank (frame 2) is given by the quaternion multiplication

$$
{ }_{2} \boldsymbol{q}_{1}=\boldsymbol{q}_{2}^{*} \otimes \boldsymbol{q}_{1}
$$

and the estimated Euler angle around yaw $\psi^{(a)}=\psi\left({ }_{2} \boldsymbol{q}_{1}^{(a)}\right)$. The angular difference between the filtered encoder measurement $\bar{\psi}$ and the estimate from the relative quaternion $\psi^{(a)}$ is given by $\Delta \psi=\bar{\psi}-\psi^{(a)}$. Subsequently, both orientation estimates are corrected by $\Delta_{2} \boldsymbol{q}$ with half the angular difference in opposite directions to retain the relative yaw angle measured by the angular encoder. Then, the correction quaternion is computed as SLERP between $\boldsymbol{q}_{I}$ and $\Delta_{2} \boldsymbol{q}$ respectively $\Delta_{2} \boldsymbol{q}^{*}$ weighted with the experimentally gathered gain $\alpha_{e}=0.5$ as in (3). Finally, the correction quaternion ${ }_{2} \boldsymbol{q}_{2}^{\text {corr }}$ is applied on $\boldsymbol{q}_{2}^{(a)}$ directly and ${ }_{2} \boldsymbol{q}_{1}^{\text {corr }}$ on $\boldsymbol{q}_{1}^{(a)}$ after transformation to frame 1 with subsequent renormalization to unit length.

Since this update is based on Euler angles, a singularity occurs when the z-axis approaches horizontal direction. Therefore, the update step is not performed when $\mathrm{z}$ is nearly horizontal $\left(60^{\circ}\right.$ to $\left.120^{\circ}\right)$ for either IMU, which is determined by

$$
q_{i 0}^{2}-q_{i 1}^{2}-q_{i 2}^{2}+q_{i 3}^{2}<0.5 \quad \forall i
$$

The relative foot sole pose is then computed with (5).

\section{B. Inertial Force Compensation}

The force measurements are compensated for inertial forces that occur due to acceleration of the mass below the force sensor. The compensation uses the measured accelerations of the foot sole IMU (IMU1) and considers the center of gravity (COG) depending on the relative foot sole pose. The mass above the pivot joint $P$ is $m_{A}=0.040 \mathrm{~kg}$ and its center of gravity has a distance to the pivot joint of ${ }_{F} \boldsymbol{r}_{P A}=$ $[0,0,0.009]^{T} m$. The mass below the pivot joint is $m_{B}=$ $0.121 \mathrm{~kg}$ with a distance of ${ }_{1} \boldsymbol{r}_{P B}=[0,0,-0.008]^{T} \mathrm{~m}$. The pivot joint has a distance to the force sensor $F$ of ${ }_{F} \boldsymbol{r}_{F P}=$ $[0,0,-0.037]^{T} m$. 


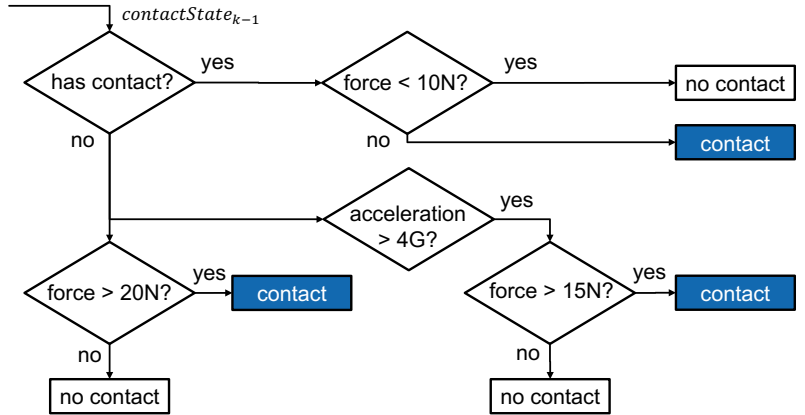

Fig. 6: Depending on the previous contact state it is either checked if the foot newly is in ground contact or if it remained in ground contact. To prevent unstable behavior of the contact estimate a hysteresis is included between detecting ground contact and detecting a loss of contact.

By neglecting the relative accelerations around the pivot joint the measured acceleration in force sensor frame is

$$
\begin{gathered}
{ }_{F} \boldsymbol{q}_{1}=\boldsymbol{q}(-\bar{\psi}) \otimes{ }_{2} \boldsymbol{q}_{1} \\
{ }_{F} \overline{\boldsymbol{a}}_{1}=\boldsymbol{R}\left({ }_{F} \boldsymbol{q}_{1}\right) \cdot{ }_{1} \overline{\boldsymbol{a}}_{1} .
\end{gathered}
$$

The relative accelerations are presumably small while the foot is in the air during swing phase. Thus, the accelerations measured by the foot sole IMU (IMU1) are transformed with the current relative pitch and roll orientation estimate to force sensor frame. The distance of both COG to the force sensor follows as

$$
\begin{aligned}
& { }_{F} \boldsymbol{r}_{F A}={ }_{F} \boldsymbol{r}_{F P}+{ }_{F} \boldsymbol{r}_{P A} \\
& { }_{F} \boldsymbol{r}_{F B}={ }_{F} \boldsymbol{r}_{F P}+\boldsymbol{R}\left({ }_{F} \boldsymbol{q}_{1}\right) \cdot{ }_{1} \boldsymbol{r}_{P B} .
\end{aligned}
$$

Then the correction forces and torques are calculated with

$$
\begin{aligned}
& \boldsymbol{F}_{\text {corr }}=\left(m_{A}+m_{B}\right) \cdot{ }_{F} \overline{\boldsymbol{a}}_{1} \\
& \boldsymbol{T}_{\text {corr }}=\left({ }_{F} \boldsymbol{r}_{F A} \cdot m_{A}+{ }_{F} \boldsymbol{r}_{F B} \cdot m_{B}\right) \times{ }_{F} \overline{\boldsymbol{a}}_{1}
\end{aligned}
$$

and applied by $\boldsymbol{F}=\boldsymbol{f}-\boldsymbol{F}_{\text {corr }}$ and $\boldsymbol{T}=\boldsymbol{t}-\boldsymbol{T}_{\text {corr }}$.

\section{Contact Detection}

The force and acceleration measurements are used to estimate if the foot is in ground contact. First, the forces and accelerations are rotated to a frame with z-axis aligned to the gravity vector and $\mathrm{x}$-axis pointing in shank forward direction such that the contact detection can be applied to the force along the gravity vector.

To detect if the foot is in ground contact the estimator shown in Fig. 6 is used and heuristically tuned. The estimator uses a hysteresis in between $20 \mathrm{~N}$ for setting the contact state to true and $10 \mathrm{~N}$ for negating the contact state to prevent an unstable estimate if in the threshold region. Additionally, a lower threshold of $15 N$ to set the contact state to true is applied if within the last ten acceleration measurements an impact of more than $4 \cdot G$ occurred.

\section{TEST SETUP}

The functionality of the adaptive foot is checked with single function tests, ground interaction tests in comparison to the single point foot design, and subsequent tests on the robot.

\section{A. Single function tests}

To test the foot sole pose estimate, the carbon tube of the foot is rigidly mounted to a test stand. The foot sole without rubber and z-damping is connected to a Dynamixel MX-64 actuator on the height of the pivot joint in y-direction of the foot. In this way, the orientation estimate of the pitch angle can be tested. To test the estimate of the roll angle, the actuator is mounted in $\mathrm{x}$-direction. To investigate the yaw estimate the actuator is centrally mounted below the foot, and the damping in $\mathrm{x}$ and $\mathrm{y}$ direction inside the actuated rim is jammed.

The sensitivity towards swinging when the foot is lifted from the ground is tested by eccentrically hanging a weight of $2.2 \mathrm{~kg}$ with a cord to the foot. When fixing the cord with an offset in y-direction the roll motion gets deflected to its maximum. Subsequently, the cord is cut and the resulting swing motion is recorded. The test is analogously conducted for pitch motion and by having the weight twisting the foot sole for yaw motion.

The force sensor accuracy and the rotation to world coordinates is verified by pressing the foot in different orientations, shown in Fig. 10, on a scale with an accuracy of $0.3 \mathrm{~g}$. Due to the limited frequency of the scale output of $4 \mathrm{~Hz}$, no fast load changes and impacts are measured.

\section{B. Ground interaction tests}

The adaptive foot is compared to the point foot in terms of traction performance and sinking depth on a variety of soils and surfaces. For the traction coefficient tests, the two feet are mounted successively on a single-feet test bench (Fig. 7). The traction coefficient is determined by calculating the relation between an incrementally applied and measured horizontal force until slipping occurs and a static vertical force. The tests are performed on Engineering Soil Simulant 2 (ES-2), which is a very fine grained/dusty soil, Engineering Soil Simulant 3 (ES-3), which is a coarse and gravelly sand, a heterogeneous soil comprised of fine to medium sized gravel, a precast concrete block, and a piece of scaffolding (Fig. 11). The soils are loosely packed and are carefully leveled before each test to create coherent initial conditions. The interaction points with the scaffold have been varied to capture the different geometric features. The sinking depth is determined by measuring the penetration distance after dropping the feet loaded with $1.4 \mathrm{~kg}$ from a defined height of $30 \mathrm{~cm}$ on the soils.

\section{Robot tests}

Finally, the foot is mounted on the robot ANYmal for validation of the contact detection algorithm as well as the overall robustness of the system. Trotting on even terrain, over loose and fixed obstacles, on a slope and special manoevers such as climbing stairs and obstacles are performed.

\section{RESULTS}

\section{A. Single function test results}

When moving the foot around single axes, the foot sole pose estimation is capable of restoring the trajectory with 


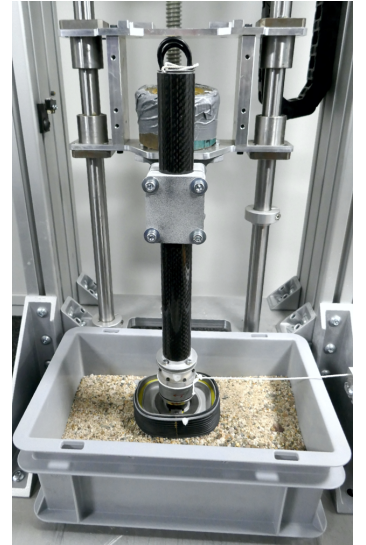

(a) Test bench setup

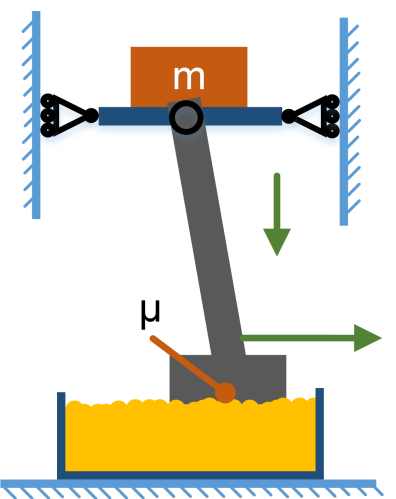

(b) Schematic test bench setup
Fig. 7: The adaptive planar foot and the point foot are mounted successively on a single-feet test bench, which allows for free linear motion in direction of gravity and one rotational degree of freedom at the mounting point. The feet are preloaded with a weight and the horizontal force to create slippage $F_{\mathrm{m}}$ is measured.

a root mean square (RMS) error of $1.24^{\circ}$ for pitch, $1.28^{\circ}$ for roll and $1.07^{\circ}$ for yaw. The trajectories are shown in Fig. 8 and it may be observed that the estimate for roll and pitch at the extremal positions diverge slightly from the actuator position. This is mainly due to reaching the end stop damping at this location with resulting deformation and high accelerations. For the estimated slope of the yaw motion, a difference of $6 \%$ to the inclination of the measured actuator motion is observed. Since this axis is determined by the accurate magnetic angular encoder it indicates that the damping in between the outer aluminum ring and foot sole basin was not completely blocked.

The swing motion resulting from returning to the initial configuration after maximum deflection for all axes is shown in Fig. 9. The swing motion decays within $0.25 \mathrm{~s}$ for all axes with the swing motion of pitch having the longest time to restore and the yaw angle not completely recovering to initial position with an offset of $0.7^{\circ}$. This is sufficient for returning to initial configurations during the leg swing phase of dynamic gaits such as trot or running trot [6]. Furthermore, it can be observed that the gyroscope of the IMU is not capable of tracking the angular velocity with its limit of $2000 \%$ at the beginning of the restoring motion. However, the decay time is identified correctly.

A comparison between the measured forces of the force/torque sensor within the foot and a scale as external reference is shown in Fig. 10. The measured forces of the force/torque sensor are rotated to a gravity aligned coordinate system resulting in a single graph that represents the forces measured by the reference independent on the orientation of the foot. As a result, the measurement of the applied force of approximately $50 \mathrm{~N}$ two times along every axis as indicated on the top of Fig. 10 provides a RMS error of $1.53 \mathrm{~N}$.

\section{B. Ground interaction test results}

The ground interaction test shows a higher average traction of the planar foot compared to the point foot (Fig. 11).
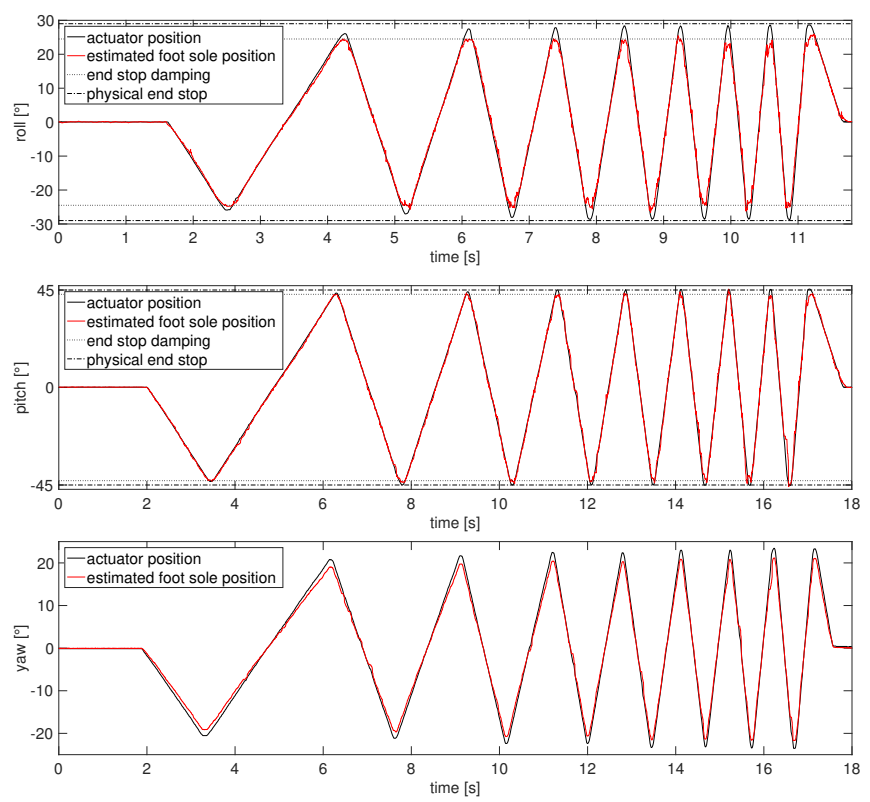

Fig. 8: The orientation estimate is able to restore the pose of the foot sole when actuated around all axis. For pitch and roll motion the estimate deviates from the actuator position after reaching the end stop damping due to the occurring high accelerations and some deformation. For yaw motion the estimate shows a slight difference of the inclination due to some remaining damping inside the actuated rim.
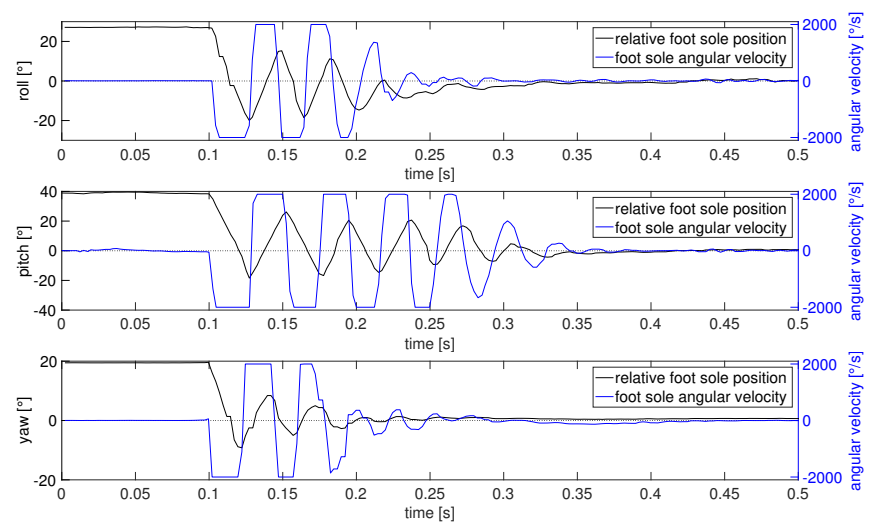

Fig. 9: The time to restore from maximal deflection back to a stable initial position is smaller than $0.25 \mathrm{~s}$ for all axes.

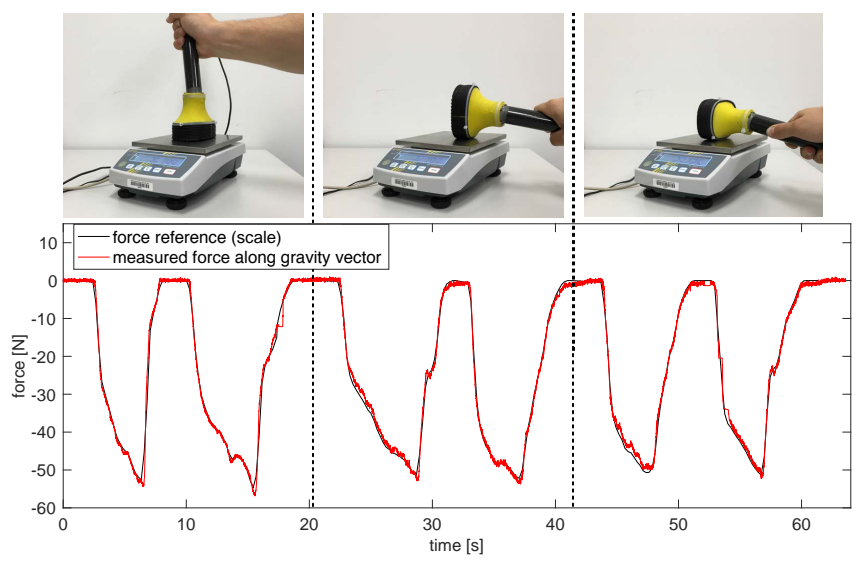

Fig. 10: The measured forces of the custom force/torque transducer are rotated to a gravity aligned frame to compare to the reference force values of a scale. The force sensor and the rotation with the orientation estimate result in a RMS of the combined measurement of $1.53 \mathrm{~N}$. 


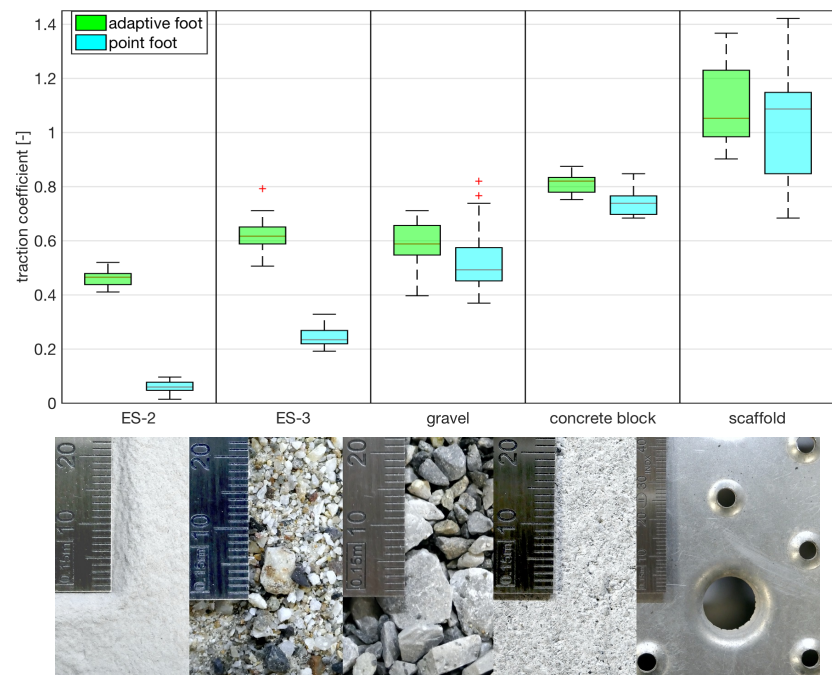

Fig. 11: The average traction coefficient is generally higher on the planar foot compared to the point foot. The difference is significant on compressible, sandy soils. A set of 30 trials is collected for each foot and terrain.

This effect is significant on the rather fine ES-2 and ES-3 soils. Observation during the experiments revealed also that on those soils, the point foot tends to slip rapidly once the point of slippage is overcome compared to the planar foot, which shows a more beneficial slip-stick behavior. Experiments on gravel show no significant advantage of the planar foot. The high variance of the point foot data on gravel is explained by the heterogeneity of the soil. However, the larger surface area of the planar foot captures more features on the soil which results in a slightly lower variance. Testing the feet on the casted concrete block does not show significant differences. The slightly higher traction of the planar foot can be explained by a better load distribution and thus lower pressure in the contact area, which is beneficial for rubber friction. Tests on the scaffold show a traction coefficient larger than one because of form fit of the feet with the geometric features of the scaffold. The high variance of the point foot is explained by the variation of the contact area during testing whereas the planar feet is able to grasp more geometrical features on average.

The sinking depth of the planar foot is generally much lower than the sinking of the point foot (Fig. 12). One can argue that a lower sinking depth is increasing agility since the feet do not get stuck, thus reduces the risk of falling, and increasing the energy efficiency of the system.

\section{Robot test results}

The contact detections based on the forces along the gravity vector and the norm of the forces both performed reliably when testing on the robot with a trotting gait as shown in Fig. 13. It is observed that the detection using the norm of the forces is more prone to measure contacts when the foot is in lift off or touch down phase resulting from measured shear forces. Sometimes, both estimates detect a short double tapping when stepping on the ground that however does not affect locomotion control.

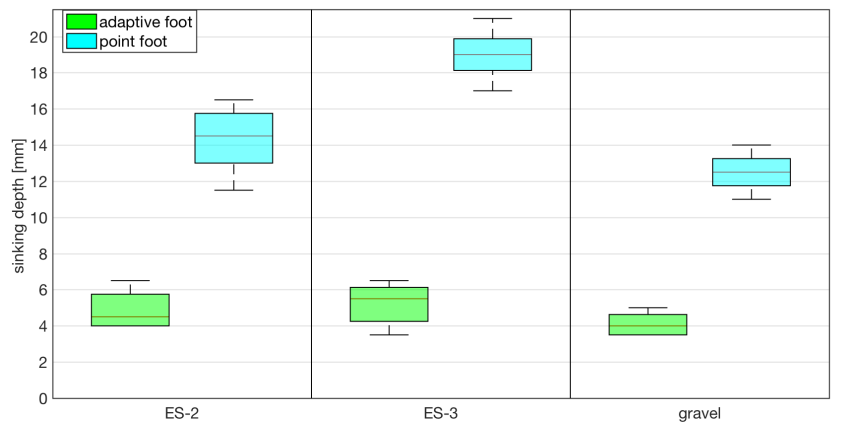

Fig. 12: The sinking depth of the planar foot is lower compared to the point foot due to the higher surface area. A set of 5 trials is collected for each foot and soil.
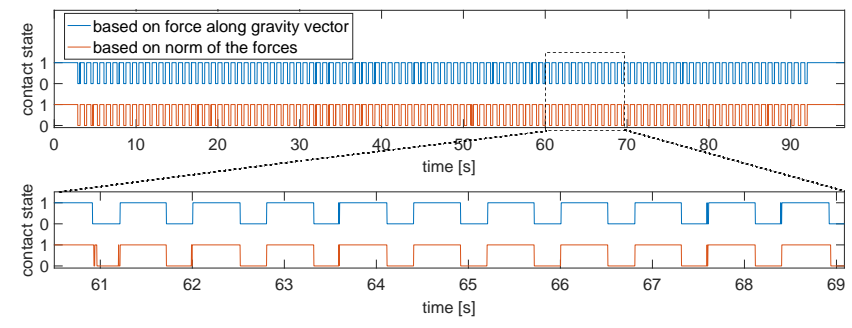

Fig. 13: The contact detection computed on the foot performs reliable when having the foot mounted on the quadrupedal robot ANYmal. It is observed that the contact estimate using the norm of the forces is slightly more prone to detect contacts when in lift-off or touch-down.

The adaptive foot allows performing all gaits the robot can perform with its ball-shaped feet. A selection of performed gaits with the foot mounted to the front right leg is shown in Fig. 14. When walking over small obstacles the foot adapts to the ground and its entire range of motion is utilized (Fig. 14a). Furthermore, the foot is capable of keeping its entire sole in ground contact every step while walking on a slope with an inclination of $19^{\circ}$ (Fig. 14b) and during obstacle or stair climbing (Fig. 14c).

\section{CONCLUSIONS}

We have presented a lightweight, adaptive planar foot that passively adapts to the ground, provides information on the ground orientation, has good traction performance especially on compressible soils, and measures forces that allow for reliable contact detection. A complementary filter to couple two IMUs with an angular encoder was implemented and successfully tested. It was shown that sensor fusion and processing can be done within the foot and thus reduces the

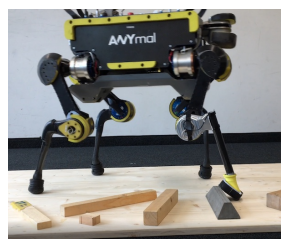

(a) trotting over obstacles

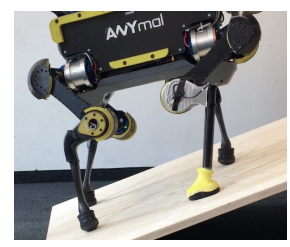

(b) walking on $19^{\circ}$ slope

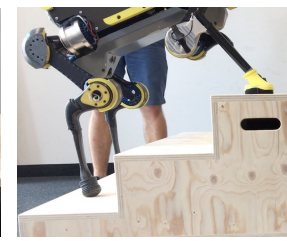

(c) stair-climbing
Fig. 14: The adaptive foot allows for performing all gaits possible with ANYmal and keeps the entire foot sole in ground contact in a large range of motion. 
computational load on the main processing unit of the robot.

The range of motion of the joint enables for $\pm 30^{\circ}$ of roll and $\pm 45^{\circ}$ of pitch adaption to the ground. Additionally, the provided yaw compliance of $\pm 20^{\circ}$ prevents the foot from slipping when turning. After maximum deflection, the foot returns to the initial position within $0.25 \mathrm{~s}$. Ultimately, it allows for the current range of motion of the robot and withstands the operational loads of all gaits tested.

In further work, the long-term robustness and the resistance towards environmental influences will be tested. Subsequently, the equipment of ANYmal with four adaptive feet is planned. It is intended to use the provided data of the foot to enhance the robot state and contact state estimation, to detail the map of the robots surrounding and even for learning to recognize different terrains.

\section{ACKNOWLEDGMENT}

The authors thank Michael Blösch for the help with the initial draft of the sensor fusion algorithm and Dario Bellicoso, Christian Gehring, Marko Bjelonic and Péter Fankhauser for their support during testing on the robot. This work has been conducted as part of ANYmal Research, a community to advance legged robotics

\section{REFERENCES}

[1] M. Hutter, C. Gehring, A. Lauber, F. Gunther, C. D. Bellicoso, V. Tsounis, P. Fankhauser, R. Diethelm, S. Bachmann, M. Bloesch, H. Kolvenbach, M. Bjelonic, L. Isler, and K. Meyer, "Anymal - toward legged robots for harsh environments," Advanced Robotics, vol. 31, no. 17, pp. 918-931, 2017.

[2] M. Hutter, R. Diethelm, S. Bachmann, P. Fankhauser, C. Gehring, V. Tsounis, A. Lauber, F. Guenther, M. Bjelonic, L. Isler, H. Kolvenbach, K. Meyer, and M. Hoepflinger, "Towards a Generic Solution for Inspection of Industrial Sites," in Field and Service Robots (FSR), 2017.

[3] M. Raibert, K. Blankespoor, G. Nelson, and R. Playter, "BigDog, the Rough-Terrain Quadruped Robot," IFAC Proceedings Volumes, vol. 41, no. 2, pp. 10822-10 825, Jan. 2008.

[4] M. P. Murphy, A. Saunders, C. Moreira, A. A. Rizzi, and M. Raibert, "The LittleDog robot," The International Journal of Robotics Research, vol. 30, no. 2, pp. 145-149, Feb. 2011.

[5] S. Seok, A. Wang, M. Y. Chuah, D. Otten, J. Lang, and S. Kim, "Design principles for highly efficient quadrupeds and implementation on the MIT Cheetah robot," in 2013 IEEE International Conference on Robotics and Automation, May 2013, pp. 3307-3312.

[6] H. Kolvenbach, D. Bellicoso, F. Jenelten, L. Wellhausen, and M. Hutter, "Efficient gait selection for quadrupedal robots on the moon and mars," 14th International Symposium on Artificial Intelligence, Robotics and Automation in Space (i-SAIRAS 2018), June 2018.

[7] L. Wagner, P. Fankhauser, M. Bloesch, and M. Hutter, "Foot contact estimation for legged robots in rough terrain," in Advances in Cooperative Robotics, July 2016, pp. 395-403.

[8] S. A. Abad, N. Sornkarn, and T. Nanayakkara, "The role of morphological computation of the goat hoof in slip reduction," in 2016 IEEE/RSJ International Conference on Intelligent Robots and Systems (IROS), Oct. 2016, pp. 5599-5605.

[9] J. H. Hicks, "The mechanics of the foot," Journal of Anatomy, vol. 87, no. Pt 4, pp. 345-357, Oct. 1953.

[10] L. Torburn, J. Perry, E. Ayyappa, and S. L. Shanfield, "Below-knee amputee gait with dynamic elastic response prosthetic feet: a pilot study." Journal of Rehabilitation Research and Development; Washington, vol. 27, no. 4, pp. 369-84, 1990.
[11] H. M. Herr and A. M. Grabowski, "Bionic anklefoot prosthesis normalizes walking gait for persons with leg amputation," Proc. $R$. Soc. B, vol. 279, no. 1728, pp. 457-464, Feb. 2012.

[12] R. D. Bellman, M. A. Holgate, and T. G. Sugar, "SPARKy 3: Design of an active robotic ankle prosthesis with two actuated degrees of freedom using regenerative kinetics," in 2008 2nd IEEE RAS EMBS International Conference on Biomedical Robotics and Biomechatronics, Oct. 2008, pp. 511-516.

[13] E. M. Ficanha, M. Rastgaar, and K. R. Kaufman, "A two-axis cabledriven ankle-foot mechanism," Robotics and Biomimetics, vol. 1, no. 1, p. 17, Nov. 2014.

[14] C. Hubicki, J. Grimes, M. Jones, D. Renjewski, A. Sprwitz, A. Abate, and J. Hurst, "ATRIAS: Design and validation of a tether-free 3dcapable spring-mass bipedal robot," The International Journal of Robotics Research, vol. 35, no. 12, pp. 1497-1521, Oct. 2016.

[15] M. Hirose and K. Ogawa, "Honda humanoid robots development," Philosophical Transactions of the Royal Society of London A: Mathematical, Physical and Engineering Sciences, vol. 365, no. 1850, pp. 11-19, Jan. 2007.

[16] K. Kaneko, K. Harada, F. Kanehiro, G. Miyamori, and K. Akachi, "Humanoid robot HRP-3," in 2008 IEEE/RSJ International Conference on Intelligent Robots and Systems, Sept. 2008, pp. 2471-2478.

[17] T. Buschmann, S. Lohmeier, and H. Ulbrich, "Humanoid robot Lola: Design and walking control," Journal of Physiology-Paris, vol. 103, no. 35, pp. 141-148, May 2009.

[18] I.-W. Park, J.-Y. Kim, J. Lee, and J.-H. Oh, "Mechanical design of humanoid robot platform KHR-3 (KAIST Humanoid Robot 3: HUBO)," in 5th IEEE-RAS International Conference on Humanoid Robots, 2005., Dec. 2005, pp. 321-326.

[19] F. Negrello, M. Garabini, M. G. Catalano, P. Kryczka, W. Choi, D. G. Caldwell, A. Bicchi, and N. G. Tsagarakis, "WALK-MAN humanoid lower body design optimization for enhanced physical performance," in 2016 IEEE International Conference on Robotics and Automation (ICRA), May 2016, pp. 1817-1824.

[20] C. Piazza, C. D. Santina, G. M. Gasparri, M. G. Catalano, G. Grioli, M. Garabini, and A. Bicchi, "Toward an adaptive foot for natural walking," in 2016 IEEE-RAS 16th International Conference on Humanoid Robots (Humanoids), Nov. 2016, pp. 1204-1210.

[21] K. Narioka, T. Homma, and K. Hosoda, "Humanlike ankle-foot complex for a biped robot," in 2012 12th IEEE-RAS International Conference on Humanoid Robots (Humanoids), Nov. 2012, pp. 15-20.

[22] K. Fondahl, D. Kuehn, F. Beinersdorf, F. Bernhard, F. Grimminger, M. Schilling, T. Stark, and F. Kirchner, "An adaptive sensor foot for a bipedal and quadrupedal robot," in 2012 4th IEEE RAS EMBS International Conference on Biomedical Robotics and Biomechatronics (BioRob), June 2012, pp. 270-275.

[23] S. O. H. Madgwick, A. J. L. Harrison, and R. Vaidyanathan, "Estimation of IMU and MARG orientation using a gradient descent algorithm," in 2011 IEEE International Conference on Rehabilitation Robotics, June 2011, pp. 1-7.

[24] J. L. Marins, X. Yun, E. R. Bachmann, R. B. McGhee, and M. J. Zyda, "An extended Kalman filter for quaternion-based orientation estimation using MARG sensors," in Proceedings 2001 IEEE/RSJ International Conference on Intelligent Robots and Systems. Expanding the Societal Role of Robotics in the the Next Millennium, vol. 4, 2001, pp. 20032011 vol.4.

[25] A. M. Sabatini, "Kalman-Filter-Based Orientation Determination Using Inertial/Magnetic Sensors: Observability Analysis and Performance Evaluation," Sensors, vol. 11, no. 10, pp. 9182-9206, Sept. 2011.

[26] R. G. Valenti, I. Dryanovski, and J. Xiao, "Keeping a Good Attitude: A Quaternion-Based Orientation Filter for IMUs and MARGs," Sensors, vol. 15, no. 8, pp. 19302-19330, Aug. 2015.

[27] T. Seel, J. Raisch, and T. Schauer, "IMU-Based Joint Angle Measurement for Gait Analysis," Sensors, vol. 14, no. 4, pp. 6891-6909, Apr. 2014.

[28] K. Shoemake, "Animating Rotation with Quaternion Curves," in Proceedings of the 12th Annual Conference on Computer Graphics and Interactive Techniques, ser. SIGGRAPH '85. New York, NY, USA: ACM, 1985, pp. 245-254. 